International Journal of Distributed and Parallel Systems (IJDPS) Vol.3, No.4, July 2012

\title{
Performance EVAluation of Radio Propagation Model For Vehicular Ad Hoc NETWORKS USING VANETMOBISIM AND NS-2
}

\author{
Ramesh C. Poonia ${ }^{1}$ and Vikram Singh ${ }^{2}$ \\ ${ }^{1}$ Research Scholar, Apaji Institute of Mathematics \& Applied Computer Technology, \\ Banasthali University, Banasthali, India. \\ rcpoonia@yahoo.com \\ ${ }^{2}$ School of Computer and Systems Science, Jaipur National University, Jaipur, India \\ vikramsingh.singh@gmail.com
}

\begin{abstract}
VANET is an higher version of Mobile Ad-hoc Network (MANET). Which protocols used in the MANET are suitable to the VANET also. Many researchers are involved in VANET field, who can provide life safety and traffic management system. In continuous growth of the vehicular industry, designing and implementing VANET is a complex and wide area of research. Thus, As we all know that these studies and practices are still simulation based because it is impracticable to use real vehicles for the large scenario in different conditions to develop new algorithms and protocols for VANET. A wireless channel is unfirm and lossy so simulators for VANET require a model of these characteristics. In this paper we have highlighted the need of adequate radio propagation models for VANET with VanetMobiSim and NS2 simulators as these factors play an important role to adopt the protocol suitable for vehicle communication.
\end{abstract}

\section{KEYWORDS}

Vehicular Ad-hoc NETwork, RPMs, Free Space Model, Two Ray Ground, Shadowing, Nakagami

\section{INTRODUCTION}

Vehicular Ad-hoc NETwork is a subset Mobile Ad-hoc NETwork. Many projects and research communities are working for VANETs. They provides safety and life saving applications. In this scenario vehicles are predicted as nodes and vehicle moves according to road topology they can't move in random direction like mobile ad hoc networks. VANET defines an intelligent way of using vehicular communication means that integrates on multiple ad-hoc networking technologies such as WiFi IEEE $802.11 \mathrm{~b} / \mathrm{g}$, WiMAX IEEE 802.16, Bluetooth, IRA, ZigBee for easy, accurate, effective and simple communication between vehicles on dynamic mobility [1]. In VANET communication vehicles acts as transceiver and also capable to send, receive and forward the information at the same time while creating a highly dynamic network [2]. This scenario works as MANETs but here vehicles are acting like computer nodes and these nodes are moving according to road topology. All vehicles have wireless communication technology and these moves according to traffic management rules. So we can say that in this case we can't study on behalf of real road scenario that means for this system, we required a simulator that should perfect for these type of study. The simulation based study is a feasible and useful

DOI : 10.5121/ijdps.2012.3415 
International Journal of Distributed and Parallel Systems (IJDPS) Vol.3, No.4, July 2012

alternative for the actual deployment. Simulations studies have to cover all the possibilities that are possible on real road topology and we can include in this study every type of scenario like city and highway [3]. For this communication we include some models these are radio propagation model. In this condition, it is essential that the simulated model is as close to reality as possible. We have to consider all the radio propagation model for vehicles communication in VANET like movement, density and velocity as well as conjunction scenario. In Figure 1 represents a VANET scenario example.

This paper is an attempt to focus the various possible radio propagation model available to simulate the VANET and we also highlighted the performance evaluation of four models that suitable in VANET scenario using VanetMobiSim and NS-2 simulators and that can help to adopt the protocols.

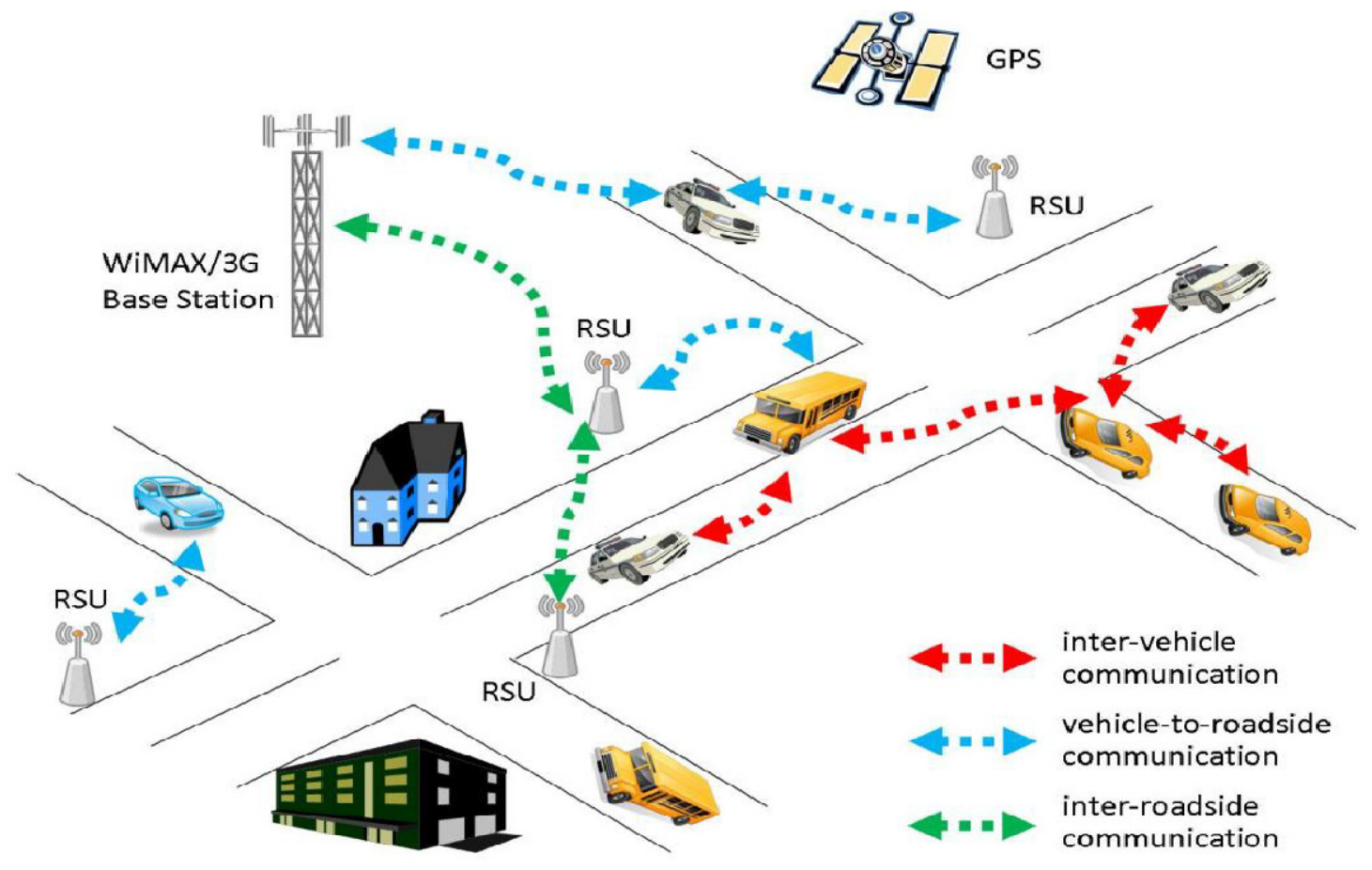

Figure 1. VANET Scenario [4]

The complete paper is organized as follows: In Section 2, we shortly provide some related work in the field of vehicular ad-hoc networks and presents the radio propagation model chosen for our simulation. Section 3 covers the overview of VanetMobiSim and NS-2 Simulators, while in Section 4, we will discuss about the radio propagation models with their properties, limitations and advantages in VANET respect and performance evolution of radio propagation models for VANET. Simulation Environment is gives in Section 5 and Experimental Analysis and Results is done in section 6 and the paper concludes in Section 7. 
International Journal of Distributed and Parallel Systems (IJDPS) Vol.3, No.4, July 2012

\section{RELATED WORK}

In this section of our paper we have mentioned some studies of radio propagation models in VANET. We found a research paper titled with Realistic Radio Propagation Models (RPMs) for VANET Simulations and written by Francisco J. Martinez, Chai-Keong Toh, Juan-Carlos Cano, Carlos T. Calafate, Pietro Manzoni [5] has evaluated different models and compared them with the Two-ray Ground model implemented in ns-2 that results obtained from simulations allow to conclude that realistic RPMs significantly affect the percentage of blind vehicles present and the number of received warning messages.

Imran Khan and others [6] has analyzed the AODV and OLSR routing protocols performances in highly fading scenario using Nakagami propagation model. A research study focused on the limitations of TwoRayGround propagation model. This study doesn't stress the details of mobility and propagation models utility.

A graduation project developed by Arijit Khan [7], title is "A comparative analysis of DSRC and 802.11 over Vehicular Ad- hoc Networks" has analyzed the performance of DSRC and 802.11 using Nakagami propagation model in city and highway for some vehicles. This study also doesn't stress the details of mobility model utility.

The paper of written by Christoph Sommer and Falko Dressler [8]. The study have well defined the historical evolution of mobility models for vehicular ad hoc networks communication. In this study has not given any importance of radio propagation model. We have also included on our paper details of mobility model defined by them.

\section{SIMULATION TOOLS}

The VanetMobiSim and NS-2 simulation tools take part in the radio propagation model for VANET simulation. Both are open source application and are express in more detail below.

\subsection{VanetMobiSim}

VanetMobiSim [9] is an extension of the CANU Mobility Simulation Environment (CanuMobiSim) [10], which focuses on vehicular mobility and features realistic automotive motion models at both macroscopic and microscopic levels. In macro-mobility takes into account the road topology and the road structure like unidirectional or bidirectional, single or multi-lane and also the road characteristics like speed limits, vehicle type and traffic signs like stop signs, traffic, lights, etc. VanetMobiSim can import maps from TIGER/Line database that support for multilane roads, separate directional flows, differentiated speed constraints and traffics signs at intersections.

The function of vehicular micro-mobility is related to all aspects of an individual vehicle like speed lane changes, acceleration and decelerations, provide communication between vehicle-tovehicle and vehicle-to-infrastructure.

\subsection{NS-2}

NS-2 [11] is mostly used for academic networking research. It is an open-source discrete event network simulator that supports both for wired and wireless networks and can simulate several network protocols such as TCP, UDP, multicast routing, etc. More recently, support has been added for simulation of large satellite and ad hoc wireless networks. The ns-2 simulation software was developed at the University of Berkeley. It is constantly under development by an active community of researchers. In this paper we have used ns-2.34 for simulation results. 
International Journal of Distributed and Parallel Systems (IJDPS) Vol.3, No.4, July 2012

Several of the mobility simulators can generate node descriptions and movement traces suitable for use in NS-2. With this couple of simulator, we will carry out an extensive performance evaluation of VANETs in terms of received packet ratio, average number of hops, average endto-end delay and data throughput, and considering several scenarios, propagation models and MAC protocols.

\section{RADIO PROPAGATION MODELS}

Radio propagation is the behavior of radio waves when they are transmitted, or propagated from one point on the Earth to another, or into various parts of the atmosphere. Like light waves, radio waves are affected by the phenomena of reflection, refraction, diffraction, absorption, polarization and scattering [12]. Below figure represents the radio propagation models and the evolution of its from free space to Nakagami radio propagation. We tried to in this paper to cover each radio propagation model in details with their suitability in VANETs.

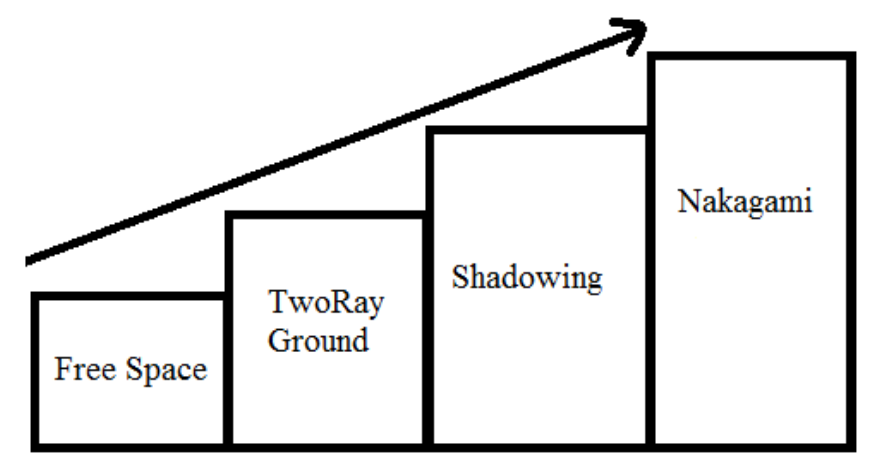

Figure 2. Radio Propagation Models Evolution for VANET

\subsection{Free Space Radio Propagation}

Free Space Radio Propagation is earliest model of radio propagation that was used in MANET by researchers. Equation 1 represents a signal propagate through open space without environmental effects. It has one parameter that is "line-of-sight". If LOS parameter is off, ground has no effect on propagation and if it is on than the model uses ground data solely to transmitting and receiving antennas [13].

$$
P(d)=\frac{P_{t} G_{t} G_{r} \lambda^{2}}{(4 \pi)^{2} d^{2} L}
$$

In above equation $\mathrm{P}(\mathrm{d})$ is the received signal power in Watt. $\mathrm{P}_{\mathrm{t}}$ is the transmitted signal power. Respectively $G_{t}$ and $G_{r}$ are the gains of the receiving and the transmitting antennas. Where $\lambda$ is the wave length, $\mathrm{L}$ is the system loss and $\mathrm{d}$ is the distance between the transmitter and the receiver.

\section{Suitability in VANET}

Free Space radio propagation model is not suitable for VANET. According to above equation $\mathrm{P}(\mathrm{d})$ is only dependent on the $\mathrm{P}_{\mathrm{t}}, \mathrm{G}_{\mathrm{t}}, \mathrm{G}_{\mathrm{r}}$ and the distance between the sender and the receiver. In VANET vehicles have to move on highway where obstacles are main concerns as the buildings alongside the road will block the communication. 
International Journal of Distributed and Parallel Systems (IJDPS) Vol.3, No.4, July 2012

\subsection{TwoRayGround Radio Propagation}

This radio propagation model is highly preferred in the research of MANET. TwoRayGround radio propagation model is also used for protocols performance in VANET scenario. This is a more realistic than the Free-Space model when we consider a ground reflected propagation path between transmitter and receiver. The model is especially useful for predicting the received power at large distances from the transmitter [14].

The received power at distance $\mathrm{d}$ is predicted by:

$$
\operatorname{Pr}(d)=\frac{P_{t} G_{t} G_{r} h_{t}{ }^{2} h_{r}^{2}}{d^{4} L}
$$

In the equation $h_{t}$ and $h_{r}$ are the heights of the transmitter and receiver antennas respectively and free space model $\mathrm{L}$ that shows a faster power loss rather than equation (1) as distance increases.

\section{Suitability in VANET}

The two-ray model does not give a good result for a short distance due to the oscillation caused by the constructive and destructive combination of the two rays. This model is not perfect for VANET.

\subsection{Shadowing Radio Propagation}

The shadowing radio propagation model have many possible scenario that can affect radio signal power The research study by Ibrahim khider and others [15] have well defined it:

The shadowing model consists of two parts. The first one is known as path loss model, which also predicts the mean received power at distance $\mathrm{d}$, denoted by $\underset{\operatorname{Pr}(d)}{\longrightarrow}$. It uses distance $\mathrm{d}_{0}$ and as a reference $\underset{\operatorname{Pr}(d)}{\longrightarrow}$ is computed relate to $\operatorname{Pr}\left(\mathrm{d}_{0}\right)$ as follows.

$$
\frac{\operatorname{Pr}(d 0)}{\operatorname{Pr}(d)}=\left(\frac{d}{d 0}\right) \beta
$$

$\beta$ is called the path loss exponent, and is usually empirically determined by field measurement. In table 1 gives some typical values of parameter $\beta$.

Table 1. Some Typical values of path loss $\beta$

\begin{tabular}{|c|l|l|}
\hline \multicolumn{2}{|c|}{ Environment } & B \\
\hline \multirow{2}{*}{ Outdoor } & Free Space & 2 \\
\cline { 2 - 3 } & Shadowing Urban Area & 2.7 to 5 \\
\hline \multirow{2}{*}{ In building } & Line-of-Sight & 1.6 to 1.8 \\
\cline { 2 - 3 } & Obstructed & 4 to 6 \\
\hline
\end{tabular}

Path loss in $\mathrm{dB}$ can be measured by given equation 4 .

$$
\left[\overline{\frac{\operatorname{Pr}(d)}{\operatorname{Pr}\left(d_{0}\right)}}\right]_{d B}=-10 \beta \log \left(\mathrm{d} / \mathrm{d}_{0}\right)
$$


International Journal of Distributed and Parallel Systems (IJDPS) Vol.3, No.4, July 2012

In the second part of the shadowing model shows the variation of the received power at assured distance. It is a lognormal random variable that is; it is of Gaussian distribution if measured in $\mathrm{dB}$. The overall shadowing model is represented by

$$
\left[\frac{\operatorname{Pr}(d)}{\operatorname{Pr}\left(d_{0}\right)}\right]_{d B}=-10 \beta \log \left(\mathrm{d} / \mathrm{d}_{0}\right)+\mathrm{X}_{\mathrm{dB}}
$$

Where $\mathrm{XdB}$ is a Gaussian random variable with zero mean and standard deviation $\sigma \mathrm{dB} . \sigma \mathrm{dB}$ is know as shadowing deviation. Table 2 shows some typical values of $\sigma \mathrm{dB}$.

Table 2. Some Typical Value of Shadowing deviation in $\mathrm{dB}$

\begin{tabular}{|l|l|}
\hline Environment & \multicolumn{1}{|c|}{$\mathbf{d B} \mathbf{~ ( d B )}$} \\
\hline Outdoor & 4 to 12 \\
\hline Office, Hard Partition & 7 \\
\hline Office, Soft Partition & 9.6 \\
\hline Factory, Line-of-Sight & 3 to 6 \\
\hline Factory, Obstructed & 6.7 \\
\hline
\end{tabular}

\section{Suitability in VANET}

The shadowing radio propagation model can be used in VANET scenario by varying either $\beta$ or $\sigma \mathrm{dB}$ values to set the various environment conditions for obstacles. This model will help to check the performance of routing protocols in VANETs for example setting $\beta$ value to 2.7 to 5 to form shadowed city scenario.

\subsection{Nakagami Radio Propagation}

This radio propagation model is well defined in [5] and given as: Nakagami is a mathematical general modeling of a radio channel with fading. With help of comparative study, we found that rather than to existing models, Nakagami model has more configurable parameters. To allows a closer representation of the wireless communication channel and it is perfect for free space channel. Nakagami distribution is defined by the following probability density function:

$$
f(x)=\frac{2 m m^{\mathrm{m}} x^{2 \mathrm{~m}-1}}{\Gamma(m) \Omega^{\mathrm{m}}} \exp \left[\frac{-m x^{2}}{\Omega}\right], x \geq 0, \Omega>0, m \geq \frac{1}{2}
$$

The corresponding pdf (probability density function) of power (square of the signal amplitude) at the given distance can be obtained by a change of variables and is given by a gamma distribution of the following form:

$$
P(x)=\left(\frac{m}{\Omega}\right)^{m} \frac{x^{\mathrm{m}-1}}{\Gamma(m)} \exp \left[-\frac{m x}{\Omega}\right],-x \geq 0
$$

$\Omega$ is the expected value of the distribution and can be interpreted as the average received power. $m$ is the so called shape or fading parameter. The values of the parameters $m$ and $\Omega$ are functions of distance. So the Nakagami model is defined by two functions:

$\Omega(\mathrm{d})$ and $\mathrm{m}(\mathrm{d})$.

- Rayleigh distribution is a special case of Nakagami distribution where $m(d)=1$ (for every $d$ ) 
International Journal of Distributed and Parallel Systems (IJDPS) Vol.3, No.4, July 2012

- Larger values of $m$ give less severe fading.

\section{Suitability in VANET}

This model is able for free space channel and moderate fading channel on highway, even to a dramatically fading channel in city scenario. By varying shape factor $m$ value we can highly fading scenario like city as well as highway. We can say that is highly applicable for protocol performance in VANETs.

\section{SIMULATION ENVIRONMENT}

The simulation model is based on VanetMobiSim and NS-2 simulation version 2.34. The simulation scenario is designed according to the normal state of vehicle running on a road shown in figure 3(a). The nodes are given in the screen scenario generator file that shows the Xfig Graph for VANETs in figure 3(b).

The generic and expected simulation process will take place in various steps discussed as follows:

- First step is installation of VanetMobiSim-1.1 and NS-2.34 and generation of traffic mobility patterns using mobility models particularly Intelligent Driving Model with Lane Changing (IDM_LC) in VanetMobiSim.

- Second step is generate the TCL file with support of mobility patterns, reliability constraints and Related Parameters.

- Third step is the trace file with help form step second will be used with macroscopic mobility model i.e. Free Space, TwoRayGround, Shadowing and Nakagami models taking one-by-one. This process will generate output files.

- And finally analyzing the generated trace file in step based on various parameters such as throughput and propagation delay with the help of graphs. Major parameters are given in table 3 for simulation.

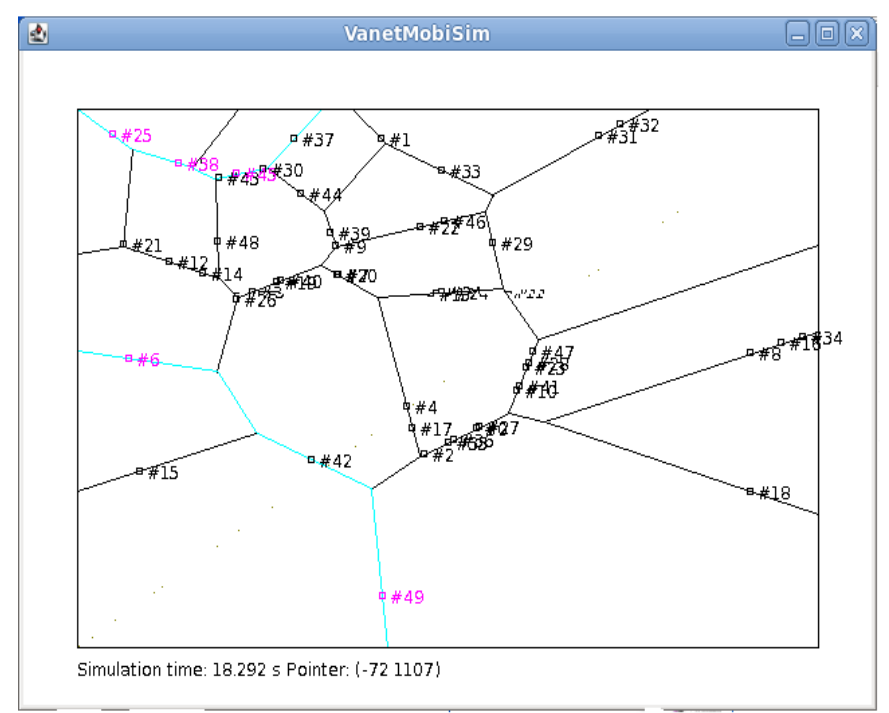

Figure 3(a). VanetMobiSim Scenario 
International Journal of Distributed and Parallel Systems (IJDPS) Vol.3, No.4, July 2012

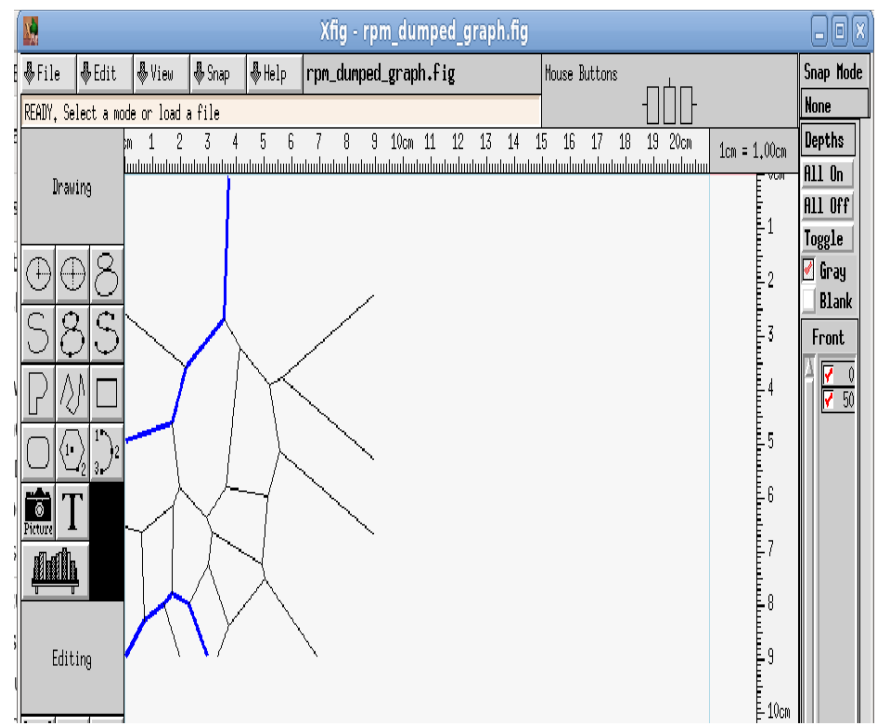

Figure 3(b). Xfig Graph of Vanet Scenario

Table 3. Simulation Parameters

\begin{tabular}{|l|l|}
\hline Parameters & Specifications \\
\hline Propagation Mode1 & $\begin{array}{l}\text { FreeSpace, TwoRayGround, } \\
\text { Shadowing, Nakagami }\end{array}$ \\
\hline Visualization Tools & VanetMobiSim, Tracing \\
\hline Network Area & $1000 \times 1000 \mathrm{~m}$ \\
\hline Radio Range & $160 \mathrm{~m}$ \\
\hline No. of Vehicle & $25,50,75,100,125,150$, \\
& 175,200 \\
\hline MAC Layer & IEEE $802.11 \mathrm{p}, 802.11$ \\
\hline Ad-hoc Routing & AODV \\
\hline Simulation Time & 100 Sec. \\
\hline Channel Type & Wireless Channel \\
\hline Traffic Type & CBR \\
\hline Min. Speed & $8.33 \mathrm{~m} / \mathrm{s}$ \\
\hline Max. Speed & $13.89 \mathrm{~m} / \mathrm{s}$ \\
\hline
\end{tabular}

\section{EXPERIMENTAL ANALYSIS}

In this section, we evaluate the impact of the RPMs with various parameters using AODV protocol. This experimental analysis discusses with the four radio propagation model for VANET implemented in NS-2.34 version.

The comparison of the four Radio Propagation Model namely Free Space, Two Ray Ground, Shadowing and Nakagami model. 
International Journal of Distributed and Parallel Systems (IJDPS) Vol.3, No.4, July 2012

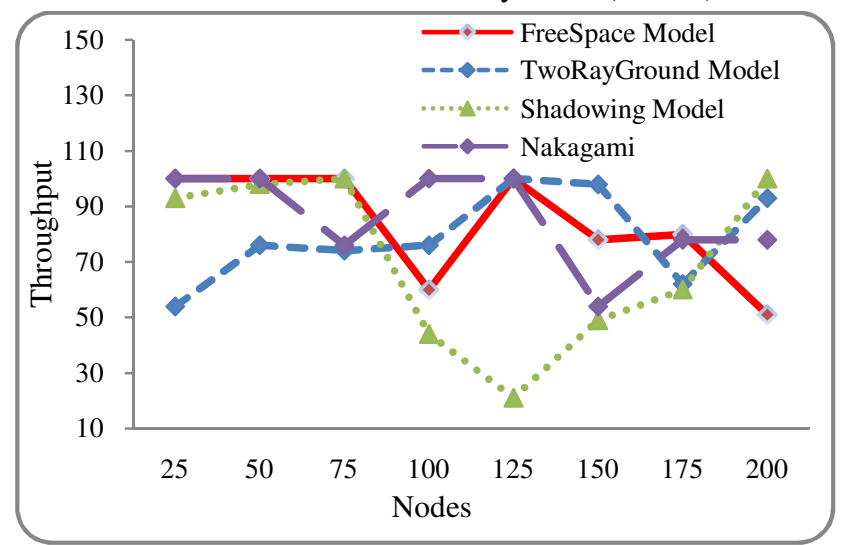

Figure 4. Throughput of various RPMs

First, we will compare four RPMs under the above simulation environment. Figure 4 and 5 shows the behaviour by throughput and packet loss of Free Space, Two Ray Ground, Shadowing and Nakagami model. We analysis that Nakagami model throughput is suitable according to no. of nodes or we can say that minimum packet loss of that propagation model.

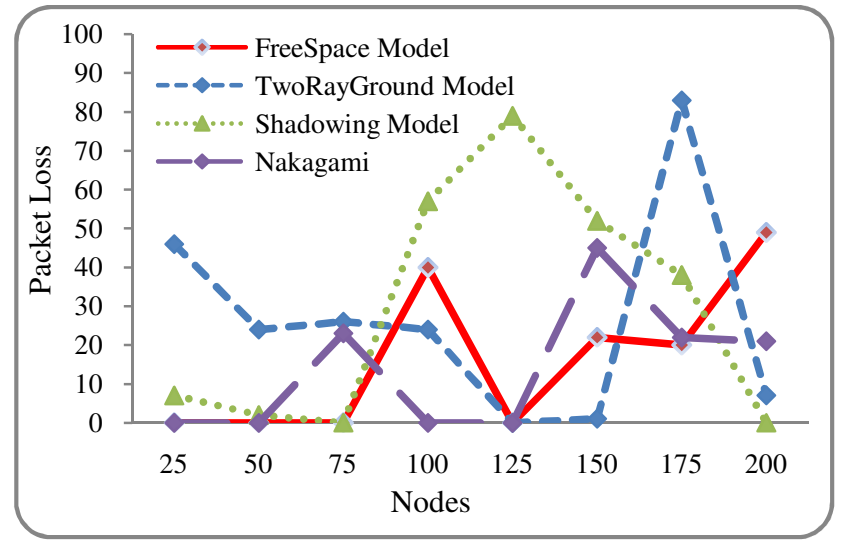

Figure 5. Packet Loss of various RPMs

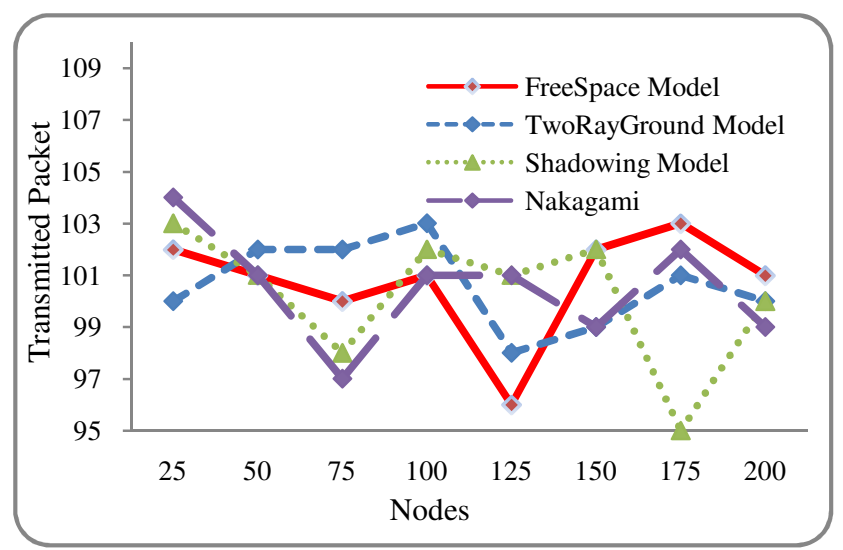

Figure 6. Transmitted Packets of various RPMs 
International Journal of Distributed and Parallel Systems (IJDPS) Vol.3, No.4, July 2012

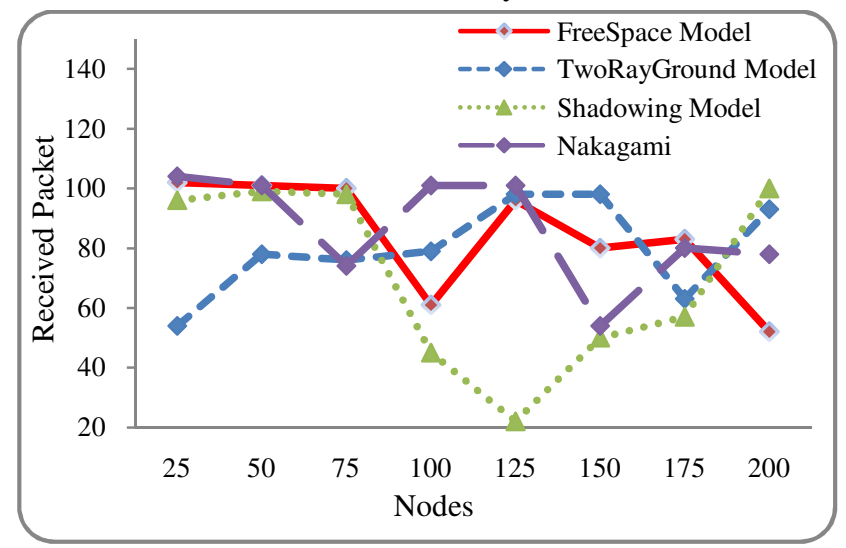

Figure 7. Received Packets of various RPMs

However, Nakagami model performs a better performance according to transmitted and received packet-wise. Shadowing model too has the worst delay characteristics because of the loss of packets information with respective nodes.

\section{CONCLUSION}

This paper has highlighted four radio propagation models and explained their features in respect to VANET. These radio propagation models are bundled with network simulator ns-2 for VANET that can help researchers to check the performance of routing protocols using these proposed and appropriate models for VANET. It will help to form realistic scenario of VANET and can be used for various applications like dangerous and accident scenario on road. We found after this study that the Nakagami is suitable and realistic radio propagation model for VANET.

\section{REFERENCES}

[1] The Theory of Vehicular Ad-Hoc Network, http://techviewz.org/2008/02/theory-of-vehicular-adhoc network.html, Visited on 18 April 2012.

[2] Jinhua Guo and Nathan Balon, "Vehicular Ad Hoc Networks and Dedicated Short-Range Communication", University of Michigan - Dearborn, June 26, 2006.

[3] Imran Khan, Master Thesis titled is "Performance Evaluation of Ad hoc Routing Protocols for Vehicular ad hoc Networks" Mohammad Ali Jinnah University, Islamabad, Feb 2009.

[4] Yue Liu, Jun Bi, Ju Yang, "Research on Vehicular Ad Hoc Networks" IEEE Chinese Control and Decision Conference", 24 June, 2010.

[5] Francisco J. Martinez, Chai-Keong Toh, Juan-Carlos Cano, Carlos T. Calafate, Pietro Manzoni, "Realistic Radio Propagation Models (RPMs) for VANET Simulations", IEEE, Wireless Communications and Networking Conference, April 2009.

[6] Khan, I. Qayyum, A. Center of Res. in Networks \& Telecom (CoReNeT), M.A. Jinnah Univ., Islamabad, Pakistan, "Performance evaluation of AODV and OLSR in highly fading vehicular ad hoc network environments" appears in Multitopic Conference, INMIC 2009, IEEE 13th, International Issue, 14-15 Dec. 2009 On page(s): 1 - 5

[7] Arijit Khan, Shatrugna Sadhu, and Muralikrishna Yeleswarapu, "A comparative analysis of DSRC and 802.11 over Vehicular Ad hoc Networks" University of California, Santa Barbara, Project of Graduation. 
International Journal of Distributed and Parallel Systems (IJDPS) Vol.3, No.4, July 2012

[8] Sommer, C.; Dressler, F.; Univ. of ErlangenNuremberg, Erlangen, "Progressing Towards Realistic Mobility Models in VANET Simulations" appears in: Communications Magazine, IEEE Issue, November 2008 ,Volume: 46 Issue:11 On page(s): 132 - 137.

[9] VanetMobiSim (Politecnico di Torino) http://vanet.eurecom.fr/

[10] CANU Research Group (Stuugart University) http://canu.informatik.unistuttgart.de/mobisim/

[11] NS-2 simulator http://nsnam.isi.edu/nsnam

[12] Pranav Kumar Singh, Kapang Lego "Comparative Study of Radio Propagation and Mobility Models in Vehicular Adhoc Network", International Journal of Computer Application, Volume 16No.8, February 2011.

[13] Mohammad Siraj \& Soumen Kanrar "Performance of Modeling wireless networks in Realistic environment" International Journal of ComputerNetworks (IJCN), Volume (2): Issue (1) 62-79

[14] Masood Khosroshahy, Thierry Turletti, Katia Obraczka, "Snapshot of MAC, PHY and Propagation Models for IEEE 802.11 in Open-Source Network Simulators", Project Report, September 2007, INRIA

[15] Ibrahim khider, Furong Wang, WeiHua Yin, Sacko, "The impact of different radio propagation models for Mobile Ad-hoc NETworks (MANET) in urban area environment" World Journal of Modelling and Simulation, Vol. 5 (2009) No.1, pp. 45-52, England, UK

\section{Authors}

Ramesh C. Poonia is research scholar of computer networking has comprehensive knowledge in the field of Information Technology. Post Master Degree competence makes him much suitable in presenting the matter in the present form. Apart from teaching currently author is pursuing Ph.D. from Banasthali University, which will open new doors for creativity in the field of Computer Science.

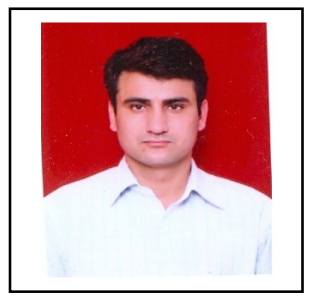

Vikram Singh is currently working as a lecturer in school of computer and system sciences at Jaipur National University, Jaipur. He has a lot of experience and knowledge in the field of computer science.

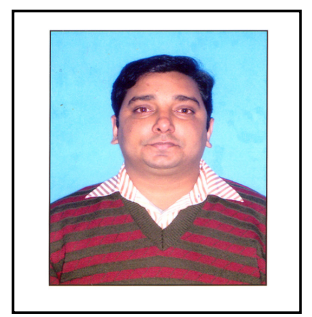

